\title{
THE
}

\section{Quantum Size Effect and the Two Types of Interference between Bulk and Boundary Scattering in Ultrathin Films}

\author{
S. Chatterjee
}

A. E. Meyerovich

University of Rhode Island, sfo101@uri.edu

Follow this and additional works at: https://digitalcommons.uri.edu/phys_facpubs

Terms of Use

All rights reserved under copyright.

\section{Citation/Publisher Attribution}

Chattejee, S., \& Meyerovich, A. E. (2011). Quantum size effect and the two types of interference between bulk and boundary scattering in ultrathin films. Phys. Rev. B, 84(16), 165432. doi: 10.1103/

PhysRevB.84.165432

Available at: http://dx.doi.org/10.1103/PhysRevB.84.165432

This Article is brought to you for free and open access by the Physics at DigitalCommons@URI. It has been accepted for inclusion in Physics Faculty Publications by an authorized administrator of DigitalCommons@URI. For more information, please contact digitalcommons-group@uri.edu. 


\title{
Quantum size effect and the two types of interference between bulk and boundary scattering in ultrathin films
}

\author{
S. Chatterjee and A. E. Meyerovich \\ Department of Physics, University of Rhode Island, Kingston, Rhode Island 02881, USA
}

(Received 11 June 2011; published 20 October 2011)

\begin{abstract}
We analyze interference between bulk and boundary scattering channels in ultrathin metal films with rough surfaces in quantum-size-effect conditions. We identify two different sources of such interference. The first, which we call the mixing interference, is associated with inversion of collision operator with surface and bulk scattering terms. This inversion always leads to mixing of bulk and surface terms, even if the scattering channels are formally independent, as long as the collision operator does not have a $\delta$-type structure (the relaxation time approximation). The second source of interference, or what we called the intrinsic interference, is associated with the physical entanglement of the scattering processes and the fact that these processes are never really independent unless both are described by the Born approximation. The intrinsic interference dominates in low-temperature clean films or when the correlation radius of surface roughness is large. The mixing interference dominates in films with robust impurity and surface scattering.
\end{abstract}

DOI: 10.1103/PhysRevB.84.165432

PACS number(s): 73.23.Ad, 73.50.Bk

\section{INTRODUCTION}

Rapid progress in material science and technology necessitated fabrication of numerous types of ultrathin conducting films and interconnects. Many of these systems exhibit a variety of quantum and surface effects. Resistivity of such conductors is an entangled combination of various surface and bulk scattering processes. The situation is further complicated because of the classical and quantum interference between different scattering channels which should be added on top of the standard approaches to transport. ${ }^{1-4}$ The aim of this paper is to try to understand the relative role played by the interference between the surface and bulk scattering in resistivity of ultrathin conductors in quantum-size-effect (QSE) conditions with noticeable quantization of electron motion. For QSE to be fully developed, it is necessary to go beyond the classical size effect that simply requires the bulk mean free path to be comparable to the film thickness.

Below we assume that the resistivity of the bulk material is known and is described by the simplest bulk collision operator. For surface contributions, we concentrate on scattering by surface roughness that can account for more than half of the overall resistivity of nanosystems. ${ }^{5}$ This assumes that the surface-driven reconstruction of the energy spectrum is less important as it is often the case in good metals. We also disregard the scattering by grain boundaries. It is well known that the presence of grain boundaries often impedes fabrication of high-quality metal films and interconnects and could be responsible for a noticeable part of resistivity and heat production (see, i.e., Refs. 6 and references therein). The presence of grain boundaries is not important when one produces films in which the size of the grains is larger than both the bulk mean free path and the film thickness (see, i.e., Ref. 7).

It is tempting to use the Mathiessen's approximation when the resistivity is due to two seemingly unrelated scattering channels such as bulk and wall scattering,

$$
\rho^{M}=\rho_{b}+\rho_{w},
$$

and the same for the inverse transport times. Here $\rho_{b}$ is the resistivity in unrestricted bulk and $\rho_{w}$ is the resistivity under the conditions when the bulk mean free path is much larger than the film thickness $L$ (we do not want to dwell here on uncertainty in ascribing the bulk parameters to the films). ${ }^{7}$ The real resistivity $\rho$ often differs significantly from the Mathiessen's value $\rho^{M}$, Eq. (1), due to various interference processes between the bulk and wall scattering channels. It is well known that the situation is even worse in the QSE conditions in which the use of the Mathiessen's equation (1) requires that both collision operators should have not just a diagonal but also the $\delta$-type structure with respect to quantized energy bands (we will encounter the clear signs of this below).

What is usually ignored is that the entanglement between the surface and bulk contributions to conductivity comes from two distinct sources. First, there is what we call here, for lack of better term, the intrinsic interference, which describes the fact that the surface and bulk collision operators $\widehat{L}_{w}$ and $\widehat{L}_{b}$ are never strictly independent of each other. The overall collision operator can be schematically written as

$$
\widehat{L}=\widehat{L}_{b}^{(0)}+\widehat{L}_{w}^{(0)}+\widehat{L}_{\mathrm{int}},
$$

where $\widehat{L}_{b}^{(0)}$ and $\widehat{L}_{w}^{(0)}$ are "pure" bulk and surface collision operators. In diagrammatic language, $\widehat{L}_{\text {int }}$ corresponds to the diagrams with the intersecting and overlapping bulk and surface interaction lines in the expansion for the Green's function while the diagrams for $\widehat{L}_{b}^{(0)}$ and $\widehat{L}_{w}^{(0)}$ contain the interacting lines of only one sort. By rearranging the terms in the diagrammatic series, it is always possible to ascribe all the intrinsic interference contributions to either one of these two collision operators while keeping the other one "pure." In what follows, we assume that $\widehat{L}_{b}$ contains only the diagrams without any surface scattering lines and is, therefore, the pure bulk collision operator, $\widehat{L}_{b}=\widehat{L}_{b}^{(0)}$. The diagrams with mixture of both bulk and surface interaction lines (the interference terms) will be taken into account by the wall collision operator $\widehat{L}_{w}, \widehat{L}_{w}=\widehat{L}_{w}^{(0)}+\widehat{L}_{\text {int }}$ and, therefore, the full collision operator

$$
\widehat{L}=\widehat{L}_{b}^{(0)}+\widehat{L}_{w} .
$$


However, even if these two operators were independent of each other (i.e., if the intrinsic entanglement could be neglected, $\widehat{L}_{\text {int }}=0$ ), there is still the second source of interference, which we will call the mixing interference. The source of this mixing can be easily understood if one considers, for example, the quantum transport equation for the density matrix (distribution function) $n$ with these supposedly independent $\widehat{L}_{w}^{(0)}$ and $\widehat{L}_{b}^{(0)}$,

$$
\frac{d n}{d t}=\left(\widehat{L}_{w}^{(0)}+\widehat{L}_{b}^{(0)}\right) n
$$

The conductivity, which is proportional to the solution of this equation, would, therefore, contain the inverse operator $\left(\widehat{L}_{w}^{(0)}+\widehat{L}_{b}^{(0)}\right)^{-1}$. It is difficult to imagine the circumstances, except for the simplest relaxation time approximations for both channels, under which this inverse operator can be decomposed into independent bulk and wall parts. This mixing, which is associated with inverting the overall collision operator, persists no matter whether we obtain the transport coefficients from solving the transport equation or from using the response function formalism such as the Kubo formulas (see, for example, Refs. 1-4). Note that the relaxation time approximation corresponds to a unique situation in which the operators $\widehat{L}_{w}^{(0)}$ and $\widehat{L}_{b}^{(0)}$ are completely independent and both have the $\delta$-type matrix structure. [Interestingly, this situation can occur in the ultraquantum regime for the QSE when all the particles are restricted to the lowest quantum miniband and the interactions do not lead to the band coupling]. To avoid mixing it is not enough for the operators to have a diagonal structure.

Most theories usually highlight just one of these two sources while neglecting the other. This often makes the comparison of the results rather meaningless. As far as we know, earlier attempts to analyze the non-Mathiessen's behavior of resistivity did not pay attention to the existence of these two different sources of interference and dealt more often than not only with the mixing interference. In those rare situations when the theoretical approaches are sufficiently sophisticated to cover the both sources, the results are somewhat convoluted and do not allow for easy interpretation and comparison with experiment. In this paper we try to bring some clarity to the picture. Below we trace both sources of interference simultaneously and analyze the conditions under which one of the interference mechanisms dominates over the other.

\section{A. Experimental background}

Before proceeding, let us comment on the possibility of observing QSE in lateral conductivity of films $\sigma$. The experimental choices for QSE measurements are rather limited: one can measure $\sigma$ as a function of roughness, bulk relaxation, or film thickness $L$. Measurements using the roughness parameters, such as the correlation radius $R$, as a (semi-) continuous variable, do not seem feasible. One can use bulk relaxation as a variable by manipulating the impurity concentration or, even better, by changing the temperature in the case of electronphonon scattering. This has its disadvantages because of an unclear link of electron-phonon relaxation in QSE conditions to the usual, relatively well-understood electron-phonon time in the bulk. Even for thicker films, away from QSE, it is not always clear what to use as the "bulk" collision time $\tau_{b}{ }^{7}$ This leaves us with measuring $\sigma(L)$.

We are interested in quasi-2D metal films with random rough surfaces. QSE is caused by quantization of motion in the direction perpendicular to the film, $p_{x} \rightarrow p_{j}=\pi j \hbar / L$, where $L$ is the film thickness, resulting in a split of the energy spectrum $\epsilon(\mathbf{p})$ into a set of minibands, $\epsilon\left(p_{x}, \mathbf{q}\right) \rightarrow$ $\epsilon(\pi j \hbar / L, \mathbf{q})=\epsilon_{j}(\mathbf{q})$. This also corresponds to slicing of a 3D Fermi surface by the planes $p_{x}=p_{j}$. Since the distance between the slicing planes $\delta p=p_{j+1}-p_{j}=\pi \hbar / L$ depends on the thickness of the film $L$, the number of slices $S$, which is equal to the integer part of $p_{F} / \delta p$,

$$
S(L)=\left\lfloor p_{F} L / \pi \hbar\right\rfloor,
$$

changes with changing $L$. Therefore, if one measures observables as a function of film thickness, one should, in principle, see some pronounced periodic singularities at the points when $S$ changes by 1 . Such QSE in metal films was observed by ultraviolet electron spectroscopy, photoemission, and various other optical methods. For our purposes, the most relevant observations use scanning tunneling microscopy (STM) and related techniques (see, for example, Refs. 8).

In a slightly different language, the changes in the number of slices of the Fermi surface by the quantizing planes $p_{x}=p_{j}$, i.e., the stepwise changes in $S(L)$, correspond to the so-called topological phase transitions. ${ }^{9}$ The most pronounced singularities associated with these continuous phase transitions, which are sometimes called the transitions of the second and a half order, appear not in thermodynamic functions, which remain continuous with continuous derivatives, but in observables most sensitive to particle scattering such as, in this case, conductivity. From purely geometrical considerations it is obvious that the ease of experimental observation of the topological changes associated with QSE depends on the shape and size of the Fermi surface and its orientation with respect to the quantizing planes (film surface). If, for example, the Fermi surface has a shape of a corrugated cylinder (open orbits), the quantizing cuts perpendicular to the cylinder axis make $S$ as a function of $L$ much larger than when the quantizing planes are directed along the axis. Large values of $S$, which are changing with much smaller increment in $L$, lead to the smearing of transitions and, essentially, to the restoration of the classical picture. From this point of view, $p_{F}$ in Eq. (5) should be understood as the size of the Fermi surface in the direction perpendicular to quantizing planes. The most advantageous situation for experiment corresponds to the smallest Fermi surfaces that are completely immersed inside the Brillouin zone such as in semimetals. In the opposite limiting case of open orbits, $p_{F}$ in Eq. (5) should be replaced by $\hbar / a_{0}$ where $a_{0}$ is the size of the crystal cell in the direction perpendicular to the film surface.

The signature feature of QSE and related topological phase transitions in metal films is a pronounced sawtooth dependence of the lateral conductivity $\sigma$ on film thickness. The sawtooth shape of the theoretical function $\sigma(L)$ has been known for decades and is common to both bulk ${ }^{10}$ and surface (roughness) $^{11}$ scattering. Still, despite numerous attempts, no one has reliably observed such a sawtooth function in good metals, that is, metals with a large Fermi surface. 
There are few observations which show promising small anomalies in $\sigma(L)$ that might indicate the presence of these, essentially quasiclassical, sawtooth singularities, ${ }^{12}$ but any positive identification of these anomalies cannot be done. The experimental reason why it is virtually impossible to observe such a sawtooth conductivity $\sigma(L)$ in metals is quite simple. The period $\pi \hbar / p_{F} \equiv \pi a$ of the sawtooth QSE oscillations in the dependence $\sigma(L)$ in good metals with large Fermi momentum $p_{F}$ is small, almost atomic, $a \sim a_{0}$. If one attempts to measure $\sigma(L)$ in films with better than atomic resolution in $L$, one would have to monitor the conductivity while gradually filling the layers. Conductivity contributions from the upper, free surface with partially filled layers with different fillings should inevitably differ from each other because of differences in scattering patterns on the partially filled surfaces. Therefore, the quasiperiodicity of $\sigma(L)$ in good conductors, even if it could be resolved, might reflect the sensitivity of scattering and, therefore, $\sigma(L)$ to layer filling rather than to QSE.

From this perspective, the more encouraging experimental objects could be semimetals with much smaller Fermi momentum $p_{F}$, such as bismuth, for which there are already some reliable claims of observing QSE singularities in conductivity of nanowires. ${ }^{13}$ Pursuing measurements in semimetals is definitely one of the experimental options. An alternative option is to look for a more recently predicted new type of QSE for films with large-scale roughness $R \gg a$. In such films, the curves $\sigma(L)$ are smooth and exhibit large-period oscillations of $\sigma(L)$ with maxima at relatively large values of $L, L_{n} \sim \pi \sqrt{n a R} \gg \sqrt{n} a$ that could lead to observation of QSE in a wider group of metals. ${ }^{14}$

At first glance, it might appear that bringing surface roughness into the picture makes revealing QSE from measuring $\sigma(L)$ in metal films not very promising. It seems impossible to maintain exactly the same surface roughness when growing the film; apart from everything else, partial filling by itself introduces atomic roughness that depends on the stage of growth. This is not so. Surprisingly, there are experimental ways for measuring the roughness-restricted conductivity $\sigma(L)$ without worrying about maintaining the same roughness when gradually growing the film and related issues. For example, it is possible to fabricate a film with rough lower surface (a rough buried interface), the roughness of which is imposed and maintained by a substrate, and an ideal upper (free) surface. The film then can be built up layer by layer without changes in the roughness of the buried interface. The conductivity can be measured each time when the free surface is again ideal (complete filling). For $\sigma(L)$ the film thickness becomes not a continuous but a discrete variable with an atomic increment. QSE in metal films with inhomogeneous buried interfaces has already been observed experimentally by STM and related techniques though without simultaneous measurements of lateral conductivity. ${ }^{8}$ Conductivity in these types of experiments can be measured using, for example, the same multiprobe $\mathrm{STM}^{13,15}$ or atomically clean nanoelectrodes. ${ }^{16}$

Note that our quantum transport theory already has an experimental confirmation in the case of ultracold neutrons in a rough waveguide. ${ }^{17}$ In this case, QSE is ensured by both the small cross section of the waveguide and the presence of the gravity field. Another experimental confirmation, which includes the study of the interference between bulk and surface scattering, is provided by recent experiments on transport in ${ }^{3} \mathrm{He}$ films, ${ }^{18}$ the theoretical interpretation of which is also based on our theory. As usual, such quantum systems are understood to be models for more complicated metal systems.

\section{B. Theoretical background}

A usual approach to transport in films is to account for bulk scattering processes via a collision operator in a transport equation and to relegate all boundary scattering to some boundary condition (for one of the best-known examples of earlier work of this type see Refs. 19). If going this route, one should express the phenomenological parameters in the boundary condition (such as, for example, the specularity coefficient $p$ or the $\mathrm{Namba}^{20}$ ratio of the amplitude of roughness and the mean free path, $\ell / \mathcal{L}_{b}$ ) via physical characteristics of surface. More sophisticated approaches with integrodifferential boundary conditions could require even more parameters in addition to the geometrical and statistical properties of surface roughness. There are two issues associated with such approaches. First, one has to postulate the form of the boundary condition. This choice of the form of the boundary condition by itself imposes limitations, which are not always clear, on what kind of surface physics can or cannot be properly incorporated into this condition. More rigorous approaches of this type require the derivation of the boundary condition. When addressing the interference between bulk and boundary scattering, this derivation and, therefore, the resulting boundary condition, should explicitly include the bulk scattering operator that makes the accurate derivation practically impossible. As far as we know, no one has performed such a derivation.

More recently, alternative approaches to boundary scattering that in clean systems can be dominated by the scattering by boundary roughness have appeared. These approaches are based on efforts to include the surface-driven distortion into the bulk Hamiltonian well beyond early attempts to artificially replace surface scattering by a set of bulk scattering centers ("impurities") located near the surface. One of the well-developed approaches of this type is a mapping transformation technique, which was suggested in Refs. 11,21, and 22 for transport in ultrathin systems. This approach involves mapping a system with random rough boundaries onto a mathematically equivalent physical system with ideal boundaries but a distorted bulk Hamiltonian. This allows one to incorporate the scattering by surface roughness inside the same collision operator as the bulk scattering processes. Other methods to include the effects of surface roughness into the bulk Hamiltonian follow the perturbative technique of Ref. 23.

The rigorous derivation of the transport equation within the mapping transformation framework and its comparison with the results based on the simplified nonunitary mapping transformation are discussed in detail in Ref. 24a. Though this is not the only approach to scattering by surface roughness (for a short review see Ref. 24b), the mapping transformation is convenient for the simultaneous description of bulk and surface scattering by providing the uniform description of all scattering channels within the single mathematical formalism. As a result, this approach naturally covers the interference non-Mathiessen's terms (see the second Ref. 21). The mapping transformation approach allows one not only to develop a 
mathematically rigorous derivation for the bulk quantum transport equation and the collision operator, which reflects the boundary roughness in the initial problem, but also to understand the limitations and accuracy of alternative approaches to the problem. ${ }^{24}$ Most of the other perturbative approaches have an accuracy similar to that of the simplified nonunitary mapping transformation. However, in contrast to the mapping transformation, there is no clear way to improve the accuracy of these approaches.

A somewhat similar, although technically different, quantum transport theory based on the surface scattering model of Ref. 23 (see also Ref. 25) has been outlined in Refs. 26 and 27 in the white-noise approximation for a rough surface (see also the extension of this approach beyond the white noise in Ref. 28). This approach corresponds to adding the surface scattering as a perturbation of the type ${ }^{23}$ to the single-particle Green's function that already includes the bulk scattering. In diagrammatic language, this corresponds to adding a surface interaction line on top of the propagator averaged over the bulk interaction (bold line). Such an approach excludes from the outset all the diagrams with the intersecting bulk and surface interaction lines, which were included in Ref. 29, and, therefore, misses some of the interference terms. Moreover, the perturbative approach of Ref. 23, is, probably, the simplest as long as it is justified, and has a narrower application domain than the properly applied mapping transformation, similar to that of the nonunitary mapping transformation (see Refs. 24).

The mapping transformation provides a theoretical framework for transport formalism for systems with differenty types of scattering channels. Since now bulk and surface scattering channels are treated in the same way, the results should reveal the full physical interference between bulk and surface scattering in transport. Based on this approach, in Ref. 29 we developed a rigorous diagrammatic derivation of the quantum transport equation for particles in quantized films with bulk and boundary scattering. Though this approach is indeed rigorous and has well-defined accuracy, the resulting equations are too complicated and difficult to use because of the interplay between numerous physical parameters and sources of interference.

Below we apply the results of our quantum diagrammatic transport derivation $^{29}$ to the analysis of non-Mathiessen's terms. We will try to cut through the parameter clutter and get results that can be used for experimental applications. We will also try to look separately at the various sources of the interference and evaluate their relative importance. Where possible, we will simplify the results in the limit of ultraclean systems with a large mean free path for which it is often possible to present the results as an expansion in inverse collision frequency.

\section{MAIN EQUATIONS}

As it is mentioned in the Introduction, QSE in films is responsible for splitting the $3 \mathrm{D}$ spectrum $\epsilon(\mathbf{p})$ into a set of $S$ minibands $\epsilon_{j}(\mathbf{q})$, where $S$ is the integer part of $L / \pi a$, Eq. (5), the atomic-size constant $a$ is equal to $a=\hbar / p_{F}$ (in semimetals $a$ is noticeably larger than atomic size), and $\mathbf{q}$ is the component of momentum along the film $\{$ in the simplest case of parabolic spectrum with the effective mass $m, \epsilon(\mathbf{p})=p^{2} / 2 m$, the minibands are also parabolic, $\epsilon_{j}(\mathbf{q})=$ $\left.(1 / 2 m)\left[(\pi j \hbar / L)^{2}+q^{2}\right]\right\}$. As a result of this quantization, all the equations acquire the matrix character in the miniband index $j$. The 2D Fermi momentum for each miniband is given by the equation $\epsilon_{j}\left(\mathbf{q}_{j}\right)=\epsilon_{F}$. For parabolic bands $q_{j}^{2}=$ $p_{F}^{2}-(\pi j \hbar / L)^{2}$. We will also introduce the "vector" $\vec{q}$ as the set of all $S$ parameters $q_{j} \equiv\left|\mathbf{q}_{j}\right|$ keeping the boldface notations for "real" $2 \mathrm{D}$ vectors $\mathbf{q}$ and $\mathbf{q}_{j}$.

After usual manipulations and expansion in harmonics, the quantum transport equation on the Fermi surface reduces to a set of linear equations

$$
\frac{q_{j}}{m}=-\sum_{j^{\prime}} \gamma_{j j^{\prime}} v_{j^{\prime}},
$$

where $v_{j}$ describes the first angular harmonic (with respect to electric field $\mathbf{E}$ ) of the deviation of the distribution function from the equilibrium $\delta f^{(1)}$,

$$
\delta f^{(1)}(\vec{q})=v_{j}(\vec{q}) \delta\left(\epsilon-\epsilon_{F}\right) e E,
$$

and the matrix $\widehat{\gamma}$ is determined by the zeroth and first harmonics of the collision vertex,

$$
\gamma_{j j^{\prime}}(\vec{q})=\delta_{j j^{\prime}} \sum_{j^{\prime \prime}=1}^{S} \Gamma^{(0)}\left(q_{j}, q_{j^{\prime \prime}}\right)-\Gamma^{(1)}\left(q_{j}, q_{j^{\prime}}\right) .
$$

In these notations, the conductivity and the transport time are

$$
\sigma=-\frac{e^{2}}{3 \hbar^{2}} \sum_{j} v_{j} q_{j}
$$

and

$$
\tau_{\mathrm{tr}}=\vec{q} \cdot \widehat{\gamma}^{-1} \vec{q} / \vec{q} \cdot \vec{q} .
$$

Note that $\sigma$ in Eq. (9) is already integrated across the film and is the $2 \mathrm{D}$ conductivity. It has the dimensionality of conductance and describes the current per unit area of the film.

In this paper we are not interested in details of the bulk collision operator that has been thoroughly studied in the literature on metals. Therefore, for the bulk-related part of the matrices $\Gamma_{j j^{\prime}}$ we will use the simplest possible expression by introducing the single bulk transport time $\tau_{\mathrm{tr}}^{b}$,

$$
\delta_{j j^{\prime}} \sum_{j^{\prime \prime}=1}^{S} \Gamma_{j j^{\prime \prime}}^{b(0)}-\Gamma_{j j^{\prime \prime}}^{b(1)}=\frac{\delta_{j j^{\prime}}}{\tau_{\mathrm{tr}}^{b}} .
$$

In contrast to the bulk scattering, for wall scattering we will use a more accurate description. Under realistic conditions, which have been analyzed in Ref. 29 in detail, the diagrammatic equations for the full single-particle Green's functions, which include averaging over both bulk and surface scattering, contain the following imaginary part in the energy denominator that we call the effective collision time $\tau_{j}(\mathbf{q})$ for particles from each miniband $\epsilon_{j}(\mathbf{q})$ (cf. Ref. 26):

$$
\begin{aligned}
\frac{1}{\tau_{j}(\mathbf{q})}= & \frac{1}{\tau_{j}^{(b)}(\mathbf{q})} \\
& +\sum_{j^{\prime}=1}^{S} \int \frac{W_{j j^{\prime}}\left(\mathbf{q}, \mathbf{q}^{\prime}\right) / \tau_{j^{\prime}}^{(b)}\left(\mathbf{q}^{\prime}\right)}{\left[\epsilon_{j^{\prime}}\left(\mathbf{q}^{\prime}\right)-\epsilon_{F}\right]^{2} / \hbar^{2}+\left[1 / 2 \tau_{j^{\prime}}^{(b)}\left(\mathbf{q}^{\prime}\right)\right]^{2}} \frac{d \mathbf{q}^{\prime}}{(2 \pi \hbar)^{2}} .
\end{aligned}
$$


Here $\tau_{j}^{(b)}(\mathbf{q})$ is the bulk relaxation time in each miniband $\epsilon_{j}$ that should be treated not as a phenomenological parameter but as the unambiguously defined imaginary part in the denominator of the single-particle Green's function for unrestricted bulk. In our context, the bulk parameters $\tau_{j}^{(b)}(\mathbf{q})$ are determined by electron-phonon or impurity scattering in the bulk and are considered known. The wall-induced transition probabilities $W_{j j^{\prime}}\left(\mathbf{q}, \mathbf{q}^{\prime}\right)$ between the states $\epsilon_{j}(\mathbf{q})$ and $\epsilon_{j^{\prime}}\left(\mathbf{q}^{\prime}\right)$ are determined by the correlation functions of surface inhomogeneities on both walls, $\zeta_{11}$ and $\zeta_{22}$, and by the interwall correlation of surface inhomogeneities $\zeta_{12} \cdot{ }^{24}$ When the metal film can be treated as a 2D square well, the equations for these transition probabilities are quite simple:

$$
\begin{aligned}
W_{j j^{\prime}}\left(\mathbf{q}, \mathbf{q}^{\prime}\right)= & \frac{\pi^{4} \hbar^{2}}{m^{2} L^{6}}\left[\zeta_{11}\left(\mathbf{q}-\mathbf{q}^{\prime}\right)+\zeta_{22}\left(\mathbf{q}-\mathbf{q}^{\prime}\right)\right. \\
& \left.+2(-1)^{j+j^{\prime}} \zeta_{12}\left(\mathbf{q}-\mathbf{q}^{\prime}\right)\right] j^{2} j^{\prime 2} .
\end{aligned}
$$

Though most of the calculations can be performed for any type of surface correlator, here in final computations we assume that the correlations of inhomogeneities on both walls are identical $\zeta_{11}=\zeta_{22}$ and Gaussian,

$$
\begin{gathered}
\zeta(\mathbf{s})=\ell^{2} \exp \left(-s^{2} / 2 R^{2}\right), \\
\zeta(\mathbf{q})=2 \pi \ell^{2} R^{2} \exp \left(-q^{2} R^{2} / 2 \hbar^{2}\right),
\end{gathered}
$$

where $\ell$ and $R$ play the role of the amplitude (height) and correlation radius (lateral size) of surface inhomogeneities and that there are no interwall correlations, $\zeta_{12}=0$. [The Gaussian peak in the $\delta$-function limit $R \rightarrow 0$ corresponds to the white-noise correlations of Refs. 21 and 26]. In practice, the correlation function of surface inhomogeneities is not always Gaussian (see Refs. 28 and 30-32 and references within). However, there are reasons to believe that the exact profile of the correlation function becomes qualitatively important only for large-scale roughness, $R \gg L .{ }^{14}$

In the end, the overall collision operator $\widehat{\gamma}$ in the transport equation (6) acquires the form

$$
\begin{aligned}
\gamma_{j j^{\prime}}= & \frac{\delta_{j j^{\prime}}}{\tau_{t r}^{b}}+\frac{\delta_{j j^{\prime}}}{\tau_{b}} \sum_{j^{\prime \prime}=1}^{S} \int \frac{q^{\prime \prime} d q^{\prime \prime}}{4 \pi} \frac{W_{j j^{\prime \prime}}^{(0)}\left(q_{j}, q^{\prime \prime}\right)}{\left[\epsilon_{j^{\prime \prime}}\left(q^{\prime \prime}\right)-\epsilon_{F}\right]^{2}+\hbar^{2} / 4 \tau_{b}^{2}} \\
& -\frac{1}{\tau_{b}} \int \frac{q^{\prime} d q^{\prime}}{4 \pi} \frac{W_{j j^{\prime}}^{(1)}\left(q_{j}, q^{\prime}\right)}{\left[\epsilon_{j^{\prime}}\left(q^{\prime}\right)-\epsilon_{F}\right]^{2}+\hbar^{2} / 4 \tau_{b}^{2}},
\end{aligned}
$$

where $W^{(0,1)}$ are the zeroth and first angular harmonics of $W\left(\mathbf{q}, \mathbf{q}^{\prime}\right)$ with respect to the angle $\theta$ between $\mathbf{q}$ and $\mathbf{q}^{\prime}$.

The poles in the surface scattering (integral) terms in Eq. (12), which are associated with bulk scattering $\mathrm{i} / \tau_{b}$, are responsible for what we called the "intrinsic interference" in the Introduction. The "mixing interference" appears later because of the need to invert the full operator $\widehat{\gamma}$ when solving Eq. (6) even though the bulk term $1 / \tau_{\mathrm{tr}}^{b}$ in the expression for the collision operator (15) has the simplest $\delta$-type form. Even if the roughness-driven scattering probabilities $W_{j j^{\prime}}$, Eq. (12), were diagonal in the miniband index $j$, there would have still been some mixing interference. The mixing interference disappears completely only if $W_{j j^{\prime}} \propto \delta_{j j^{\prime}}$.

Note that the bulk parameters $\tau_{\mathrm{tr}}^{b}$ and $\tau_{b}$ in Eq. (15) could differ significantly: One represents the bulk transport time (the pole in the two-particle propagator) and the other the collision time (the pole in the single-particle Green's function). Below we introduce parameter $\alpha$ for the ratio of these two times,

$$
\frac{1}{\tau_{\mathrm{tr}}^{b}}=\frac{\alpha}{\tau_{b}}
$$

If the bulk scattering is associated mostly with impurities, then $\alpha$ is a number of the order of 1 . If, however, the main bulk collision channel is the electron-phonon scattering, then parameter $\alpha$ is small when the temperature is noticeably below the Debye temperature $T_{D}{ }^{4,33}$ :

$$
\alpha \propto\left(T / T_{D}\right)^{2} \ll 1 .
$$

Another important issue is that extracting the appropriate values of the bulk times $\tau_{b}, \tau_{\mathrm{tr}}^{b}$ for use in thin films is not trivial. For example, there are experimental indications ${ }^{34}$ that the presence of film surfaces leads to a noticeable softening of phonon modes and, therefore, lowering of the Debye temperature and renormalization of the electron-phonon times.

Earlier, ${ }^{35}$ instead of inverting the collision operator [Eqs. (6) and (15)], we approximated the effect of collisions on transport by inserting a heuristic factor $(1-\cos \theta)$, which leads to a proper combinations of harmonics, into the integrand and replacing summation by the integration. The resulting quasiclassical transport time provided a good estimate for what we call here the non-Mathiessen's intrinsic interference terms but did not include the mixing interference. Note that such intrinsic interference has also been investigated in various contexts in Refs. 36-38, also on the basis of our Eq. (12), without inverting the collision operator.

\section{RESULTS}

Below we will take QSE into account and perform an accurate inversion of the collision operator. One of the main feature of QSE is the sawtooth dependence of the conductivity of films on film thickness $\sigma(L)$ that, in principle, should be observed for both bulk ${ }^{10}$ and surface ${ }^{11}$ scattering. The reason is that the number of minibands $S$ increases by 1 each time the film thickness increases by $\delta L=\pi \hbar / p_{F}$. The change in $S$, by itself, should lead just to kinks on the curve $\sigma(L)$; what leads to a sharp drop in $\sigma$ and, therefore, to the sawtooth picture is the opening of a large number $S$ of new scattering channels each time $S$ changes by 1 . This means that the sawtooth dependence exists only as long as there are robust interband transitions that require the presence of noticeable off-diagonal elements in the matrix $\widehat{\gamma}$, Eq. (15). Since in this paper the bulk part of the collision operator is approximated by a diagonal matrix, the sawtooth structure of the conductivity curve originates from the surface part of the collision operator.

We will analyze the relative contributions of intrinsic and mixing interference terms. Note that the mixing interference requires the non- $\delta$-type structure of the collision operator and is, therefore, associated with the sawtooth picture of QSE in conductivity of ultrathin films. In principle, the inversion of the collision operator [Eqs. (6) and (15)], is a straightforward numerical task, especially because we are interested in thin films for which the number of minibands and, therefore, the rank of the matrix $\widehat{\gamma}$ are relatively low. The real difficulty here is not in solving the quantum transport equation numerically 
but in making sense of the results because of a large number of different parameters, for many of which the experimental information is rather sparse. Thus one of the main goals is to cut through the parameter clutter and get physically meaningful results. Let us start from the list of relevant dimensionless parameters.

The interplay between bulk and roughness scattering can be described by two dimensionless parameters, $t$ and $u$, the first of which characterizes the bulk scattering and the second the correlation of surface roughness:

$$
t=\tau_{b} p_{F}^{2} / m \hbar, u=p_{F}^{2} R^{2} / \hbar^{2} \equiv R^{2} / a^{2} \gtrsim 1,
$$

where $p_{F}$ is the Fermi momentum and $a$, defined as $a=\hbar / p_{F}$, is of the order of the atomic size. It is often convenient to describe transport in films with large bulk mean free paths as an expansion in $1 / t$. On the other hand, parameter $u$ accounts for the effectiveness of surface scattering in which the relative change in momentum is $\delta q / q \sim 1 / \sqrt{u}$. The high-quality samples are the ones in which both $t$ and $u$ are large.

Apart from $t$ and $u$, other important dimensionless parameters include $\alpha$, which characterizes the difference between the bulk collision and transport times (16), the dimensionless thickness of the film $L / a$, and the amplitude of roughness $\ell / a$. In these notations, the bulk mean free path $\mathcal{L}_{b}$ is

$$
\mathcal{L}_{b} / a=\tau_{\text {tr }}^{b} v_{F} / a=t / \alpha .
$$

With so many parameters, none of which reduces to a simple scaling, one should find a way to understand the hierarchy of competing effects that would allow one to get some clarity.

Nevertheless, one of the parameters $(\alpha$ or $\ell / a)$ can still be excluded by a proper choice of variables leaving behind only the ratio

$$
\theta=\frac{\alpha a^{2}}{\ell^{2}}
$$

To achieve this, we will describe the relative interference contribution to the resistivity $\rho$ and transport time $\tau_{\text {tr }}$ by parameter $\chi$,

$$
\chi=\frac{\rho-\rho^{M}}{\rho^{M}} \equiv \tau_{\mathrm{tr}}^{M}\left(\frac{1}{\tau_{\mathrm{tr}}}-\frac{1}{\tau_{\mathrm{tr}}^{M}}\right),
$$

where the Mathiessen's resistivity $\rho^{M}$ and transport time $\tau_{\mathrm{tr}}^{M}$ are defined as

$$
\rho^{M}=\rho_{b}+\rho_{w}, 1 / \tau_{\mathrm{tr}}^{M}=1 / \tau_{\mathrm{tr}}^{b}+1 / \tau_{\mathrm{tr}}^{w},
$$

and indices $b$ and $w$ refer to the pure independent bulk and wall contributions.

The collision operator $\gamma_{j j^{\prime}}$, Eq. (15), contains two terms. The bulk one is proportional to $\alpha$ and the wall one to $\ell^{2} / a^{2}$. Instead of $\gamma_{j j^{\prime}}$, we introduce the matrix

$$
\tilde{\gamma}_{j j^{\prime}}=\frac{a^{2}}{\ell^{2}} \gamma_{j j^{\prime}}
$$

which depends on $\alpha$ and $\ell^{2} / a^{2}$ only in the combination $\theta$ [Eq. (20). This means that the solution $\widetilde{v}_{j}=\left(\ell^{2} / a^{2}\right) v_{j}$ of the renormalized equation (20) will also depend only on $\theta$. The same will be true for the renormalized conductivity, $\widetilde{\sigma}=\sigma\left(\ell^{2} / a^{2}\right)$, resistivity $\tilde{\rho}=\rho\left(a^{2} / \ell^{2}\right)$, and the inverse transport time, $1 / \widetilde{\tau}_{\mathrm{tr}}=\left(a^{2} / \ell^{2}\right) / \tau_{\mathrm{tr}}$. Then the relative interference contribution (21),

$$
\chi=\frac{\rho-\rho^{M}}{\rho^{M}} \equiv\left(\frac{1}{\widetilde{\tau}_{\mathrm{tr}}}-\frac{\theta}{\tau_{b}}-\frac{1}{\tilde{\tau}_{\mathrm{tr}}^{w}}\right) /\left(\frac{\theta}{\tau_{b}}+\frac{1}{\widetilde{\tau}_{\mathrm{tr}}^{w}}\right),
$$

will also contain $\alpha$ and $\ell$ only in the form of the combination $\theta$. The pure roughness-driven transport time $\tilde{\tau}_{\mathrm{tr}}^{w}$ in Eq. (24) should be calculated using the surface scattering probabilities $W_{j j^{\prime}}$ [Eqs. (13) and (14)] without the factor $\ell^{2} / a^{2}$ (and, of course, in the limit $\left.\tau_{b} \rightarrow \infty\right)$.

Note that parameter $\theta / t$ provides one with an estimate for comparative contributions of surface and bulk scattering channels to transport, $\tau_{\mathrm{tr}}^{w}$ and $\tau_{\mathrm{tr}}^{b}$. However, this estimate is not very accurate because of a strong dependence of $\tau_{\mathrm{tr}}^{w}$ on $L / a$ and $u$, Eq. (18). The ratio $\tau_{\mathrm{tr}}^{b} / \tau_{\mathrm{tr}}^{w}$ is best characterized by the scaled function $\phi(L / a, u)$,

$$
\frac{\tau_{\mathrm{tr}}^{b}}{\tau_{\mathrm{tr}}^{w}}=\frac{t}{\theta} \phi(L / a, u)
$$

which depends only on $u$ and $L / a$. The function $\phi(L / a)$,

$$
\begin{gathered}
\phi=\frac{\mathbf{q} \cdot \mathbf{q}}{\mathbf{q} \cdot \widehat{F}^{-1} \mathbf{q}}, \\
F_{j j^{\prime}}=4 \pi^{5}\left(\frac{L}{a}\right)^{6} j^{2} u e^{-u} \exp \left(c_{j} / 2\right) f_{j j^{\prime}}, \\
f_{j j^{\prime}}=\delta_{j j^{\prime}} \sum_{j^{\prime \prime}}\left[j^{\prime \prime 2} \exp \left(c_{j^{\prime \prime}} / 2\right) I_{0}\left(u \sqrt{1-c_{j} / u} \sqrt{1-c_{j^{\prime \prime}} / u}\right)\right] \\
-j^{\prime 2} \exp \left(c_{j^{\prime}} / 2\right) I_{1}\left(u \sqrt{1-c_{j} / u} \sqrt{1-c_{j^{\prime}} / u}\right) \\
c_{j}=\pi^{2} u\left(\frac{a}{L}\right)^{2} j^{2},
\end{gathered}
$$

is presented in Fig. 1 as a function of $L / a$ for several values of $u=p_{F}^{2} R^{2} / \hbar^{2}, u=1,5,10,100$. Note that the thickness of the film, $L / a$, enters the expression for $\phi$ [Eq. (26)], not only via the explicit coefficient in $\widehat{F}$ but also through the upper limit $S$ of summation over $j, j^{\prime}, j^{\prime \prime}$ which depends on $L$ explicitly [Eq. (5)]. It is clear that even the pure wall contribution is comparable to the bulk term at a value for $\alpha$ that is not very small only for relatively thin films. This means that the

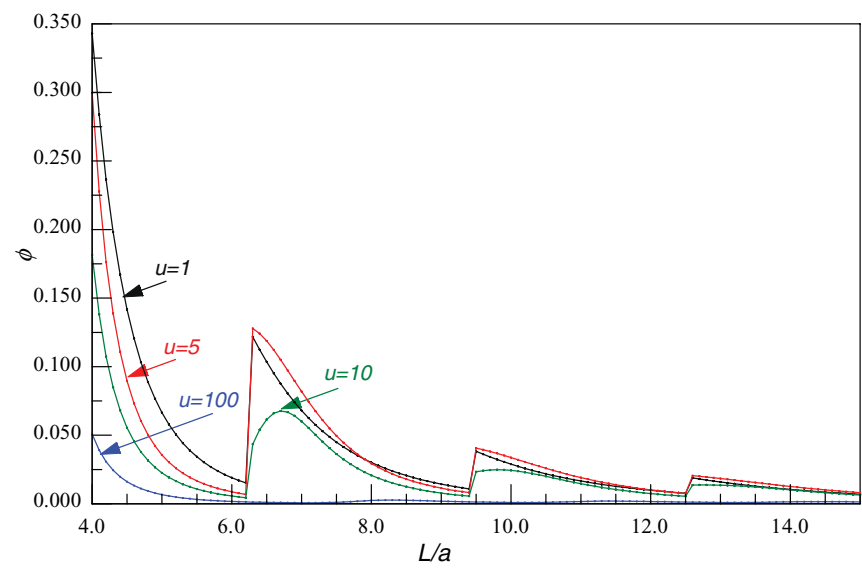

FIG. 1. (Color online) Function $\phi(L / a)$ [Eqs. (25) and (26)] for four values of $u: u=1$ (black), 5 (red), 10 (green), and 100 (blue). The curves are marked accordingly. 
interference terms, which are usually small in comparison with both pure surface and bulk contributions, should be investigated for relatively thin films for which the pure surface contribution is not negligible in comparison with the bulk one, i.e., the function $\phi(L / a, u)$ is not exceedingly small.

Figure 1 confirms that the surface contribution decreases with increasing $u$ when the film surface becomes more and more smooth. The second observation is that the increase in $u$, i.e., in $R / a$, results in smoothing of the sawteeth at $L / a=\pi j$, which corresponds to the usual QSE and leads eventually to the appearance of a new type of QSE in accordance with Ref. 14.

As indicated above, one of the main issues when dealing with the interference between the bulk and surface scattering is to separate the sources of intrinsic and mixing interference. We continue this section with an analysis of the pure intrinsic interference. This is a reasonable approximation for pure films at low temperatures for which $\alpha \ll 1$ [Eq. (17)]. After that, we will analyze the pure mixing interference by assuming $\tau_{b} \rightarrow \infty$ in the integral term only in Eq. (15). As we will see, this approximation might work reasonably well for films with the dominant impurity scattering in the bulk. Toward the end of the section, we will look at general results with both types of interference and will try to compare their contribution under various conditions.

\section{A. The intrinsic interference}

In this subsection we will look at the pure intrinsic interference. There are two different sets of conditions under which the mixing interference becomes negligible. The first is, of course, the case of the vanishingly small value of $\alpha$. This situation occurs in ultraclean films at low temperatures when the bulk scattering is dominated by longwave phonons, making the ratio of bulk collision and transport times $\alpha$ very small [Eq. (17)]. In this case one can simply put $\alpha=0$ in the collision operator [Eqs. (15) and (16)]. Since in this case the bulk transport time and conductivity are negligible by design, the relative intrinsic interference contribution (21) reduces to

$$
\chi_{\text {intr }}=\frac{\rho(\alpha=0)-\rho_{w}}{\rho_{w}} \equiv \tau_{\mathrm{tr}}^{w}\left[\frac{1}{\tau_{\mathrm{tr}}(\alpha=0)}-\frac{1}{\tau_{\mathrm{tr}}^{w}}\right],
$$

where, as above, $\rho_{w}$ and $\tau_{\mathrm{tr}}^{w}$ are the pure wall-driven resistivity and transport time in the absence of bulk scattering [i.e., the resistivity and transport time generated by the collision operator (15) with $\alpha=0$ and $\tau_{b} \longrightarrow \infty$ ]. Since here we assume from the start that $\alpha=0$, there is no need to replace the transport times $\tau$ in the right-hand side of Eq. (27) by their renormalized values as in Eq. (24); the parameter $\ell^{2} / a^{2}$ will get canceled automatically.

In this case, the only remaining parameters in $\chi_{\text {intr }}$ are $t, u$, and $L / a$. In high-quality films, parameter $t$ (i.e., $\tau_{b}$ ) is large, and it is often convenient to expand the integrand in Eq. (15) in $1 / t$ using

$$
\begin{aligned}
& \frac{1 / x \pi}{\left[\epsilon\left(\mathbf{p}^{\prime}\right)-T_{F}\right]^{2}+1 / x^{2}} \\
& =\delta\left[\epsilon_{j^{\prime}}\left(q^{\prime}\right)-T_{F}\right]+\frac{1}{x} \frac{d \delta\left[\epsilon_{j^{\prime}}\left(q^{\prime}\right)-T_{F}\right]}{d \epsilon} \\
& \quad+\frac{1}{2 x^{2}} \frac{d^{2} \delta\left[\epsilon_{j^{\prime}}\left(q^{\prime}\right)-T_{F}\right]}{d \epsilon^{2}}+\cdots .
\end{aligned}
$$

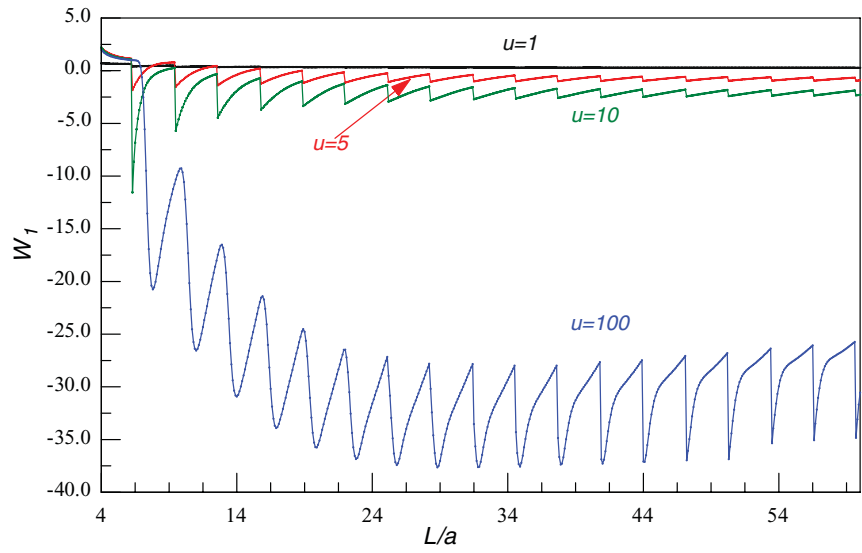

FIG. 2. (Color online) Coefficient $W_{1}$ [Eq. (29)] as a function of the film thickness $L / a$ for four values of $u: u=1$ (black), 5 (red), 10 (green), and 100 (blue). The curves are marked accordingly.

The first, $\delta$-function term in this expansion in the collision operator eventually generates the pure wall-driven resistivity and transport time $\rho_{w}$ and $\tau_{\mathrm{tr}}^{w}$. The relative interference contribution $\chi_{\text {intr }}$ then becomes

$$
\chi_{\text {intr }}=\frac{1}{t} W_{1}(u, L / a)+\frac{1}{t^{2}} W_{2}(u, L / a)+\ldots .
$$

The plots of functions $W_{1,2}(L / a)$ for several values of $u$ $(u=1,5,10,100)$ are given in Figs. 2 and 3 . The figures indicate that the relative interference contributions to the resistivity are almost always negative in the case of pure intrinsic interference except for extremely thin films (cf. Ref. 35). Another observation is that the intrinsic interference at large $u$ loses the sawtooth structure of the usual QSE and acquires the smooth QSE of Ref. 14; the sawteeth reappear for thicker films.

A more important observation concerns slightly thicker films than the ones in Figs. 2 and 3. With further increase in thickness, the values of coefficients $W_{1}$ for all values of $u$ and $W_{2}$ for smaller $u$ remain more or less the same while $W_{2}(u=100)$ rapidly increases by an order of magnitude (on this scale, the values of $W_{2}$ for $u=1,5,10$ are practically

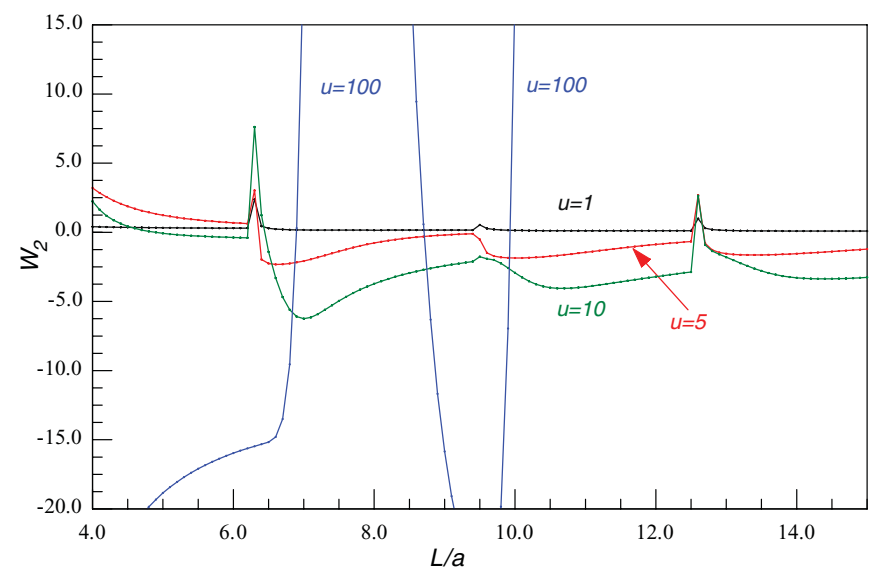

FIG. 3. (Color online) Coefficient $W_{2}$ [Eq. (29)] as a function of the film thickness $L / a$ for four values of $u: u=1$ (black), 5 (red), 10 (green), and 100 (blue). The curves are marked accordingly. 


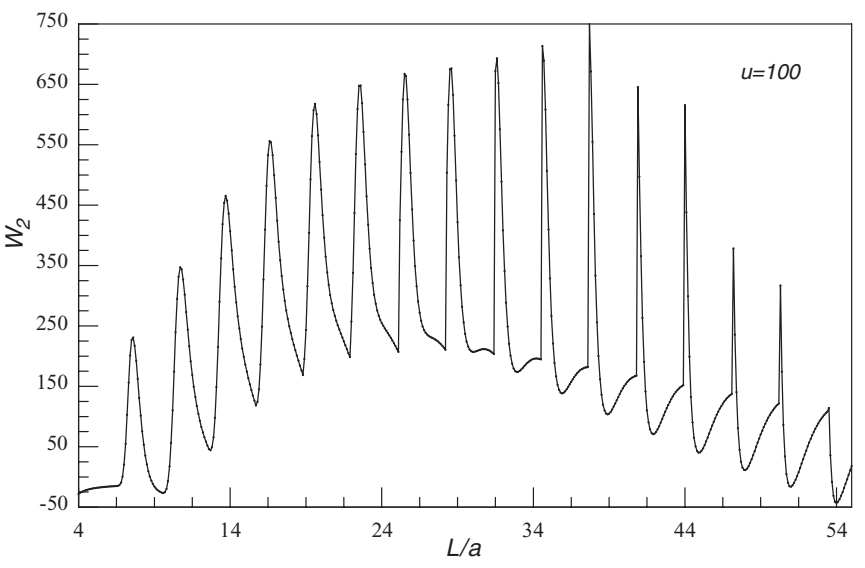

FIG. 4. Coefficient $W_{2}$ [Eq. (29)] as a function of the film thickness $L / a$ at $u=100$ for thicker films. On this scale the curves $W_{2}(L / a)$ at $u=1,5,10$ are practically unnoticeable. The curve also illustrates the smooth QSE of Ref. 14 for thin films and the restoration of the ususal sawtooth QSE for thicker films.

unnoticeable; Fig. 4). This means that in high-quality films with large values of $t$ and $u$ the intrinsic interference should experience a crossover from $1 / t$ to $1 / t^{2}$ behavior at largeenough values of $u$. Earlier we observed a similar crossover in our quasiclassical description of the intrinsic interference in Ref. 35. In accordance with Ref. 14, one sees a restoration of the usual sawtooth QSE for thicker films for which $L / a \gg$ $R / a=\sqrt{u}$.

The second situation with the dominant intrinsic interference is, with certain caveats, the case of a diagonal matrix $\widehat{\gamma}$, Eq. (15), i.e., the diagonal matrix of roughness-driven scattering probabilities $W_{j j^{\prime}}$, [Eqs. (13) and (14)]. This can happen for large-scale roughness, $u=R^{2} / a^{2} \gg L^{2} / a^{2}$, when the scattering-driven change in the particle momentum $\delta q \sim$ $\hbar / R$ is insufficient for the particle transition between the minibands, $\delta q / q \sim 1 / \sqrt{u} \ll 1$. When the collision operator $\widehat{\gamma}$ is diagonal,

$$
\gamma_{j j^{\prime}}=\gamma_{j} \delta_{j j^{\prime}}
$$

the solution of the transport equation (6) is trivial,

$$
\frac{q_{j}}{m}=-\sum_{j^{\prime}} \gamma_{j j^{\prime}} v_{j^{\prime}}, v_{j}=-q_{j} \gamma_{j}^{-1} / m,
$$

and the full transport time $\tau_{\text {tr }}$ [Eq. (10)], is quite simple:

$$
1 / \tau_{t r}=\sum q_{j}^{2} / \sum q_{j}^{2} \gamma_{j}^{-1}
$$

Since the wall contributions to $\gamma_{j}$ differ while the bulk ones are the same, $\alpha / \tau_{b}$, Eq. (31) still contains noticeable mixing terms. However, the structure of the roughness-driven scattering probabilities $W_{j j^{\prime}}\left(\mathbf{q}_{j}-\mathbf{q}_{j^{\prime}}^{\prime}\right)$ [Eqs. (13) and (14)] is such that the diagonal terms $W_{j j}$ differ from each other by the factors $j^{4}$. Since $q_{j}^{2}$ decrease with increasing $j$, the overall transport time $1 / \tau_{\text {tr }}$, Eq. (31), can with good accuracy be rewritten as

$$
1 / \tau_{\mathrm{tr}} \approx \frac{\gamma_{1}}{q_{1}^{2}} \sum q_{j}^{2}=\frac{1}{q_{1}^{2}}\left(\frac{\alpha}{\tau_{b}}+\frac{1}{\tau_{11}^{w}}\right) \sum q_{j}^{2},
$$

where $1 / \tau_{11}^{w}$ is the integral term with $W_{11}$ in the expression for $\gamma_{j j^{\prime}}$, Eq. (15). The approximation (32) emphasizes the dominant role of gliding particles in the lateral transport in quantized ultrathin films and contains only the intrinsic interference.

\section{B. The mixing interference}

The mixing interference can be obtained by formally putting $\tau_{b} \longrightarrow \infty$ in the wall-driven part of the collision operator (15). Then the bulk relaxation time enters $\chi_{\text {mix }}$ [Eqs. (21) and (24)] only in the combination $\theta / t$,

$$
\chi_{\operatorname{mix}}=\chi_{\operatorname{mix}}(\theta / t, u, L / a) .
$$

Unfortunately, the structure of $\chi_{\text {mix }}$ is such that all its variables $(\theta / t, u$, and $L / a)$ enter the equations independently and none of the variables reduce to simple scaling of $\chi_{\text {mix }}$ or can be eliminated.

One should be very cautious when inverting the collision operator numerically. The roughness-driven part of the collision operator (15) was calculated in Ref. 29 only to the main order in the roughness amplitude $\ell$. Therefore, many higher-order terms in $\ell^{2}$, which arise from the inversion of the collision operator, should be disregarded.

To solve the transport equation and, therefore, calculate the transport time, one should simply invert the collision operator (matrix) $\tilde{\gamma}_{j j^{\prime}}$. The wall-driven part of the matrix $\tilde{\gamma}_{j j^{\prime}}$ [Eq. (15)] in the limit $\tau_{b} \longrightarrow \infty$ corresponds to the first, $\delta$-type, term in expansion (28) and becomes relatively simple. Using the notations of Ref. 14 , the matrix $\tilde{\gamma}_{j j^{\prime}}$ can then be rewritten as

$$
\begin{gathered}
\tilde{\gamma}_{j j^{\prime}}=\frac{a^{2}}{\ell^{2}} \gamma_{j j^{\prime}}, \gamma_{j j^{\prime}}=\frac{\alpha \delta_{j j^{\prime}}}{\tau_{b}}+\frac{1}{\tau_{j j^{\prime}}}, q_{j} / m=-\sum_{j^{\prime}} v_{j^{\prime}}\left(q_{j^{\prime}}\right) / \tau_{j j^{\prime}} \\
\frac{1}{\tau_{j j^{\prime}}}=\frac{m}{2} \sum_{j^{\prime \prime}}\left[\delta_{j j^{\prime}} W_{j j^{\prime \prime}}^{(0)}-\delta_{j^{\prime} j^{\prime \prime}} W_{j j^{\prime}}^{(1)}\right]
\end{gathered}
$$

In the case of the Gaussian correlation functions, the harmonics of the scattering probabilities $W$ are given as

$$
\begin{gathered}
\zeta^{(0)}\left(q_{j}, q_{j^{\prime}}\right)=4 \pi \ell^{2} R^{2}\left[e^{-Q Q^{\prime}} I_{0}\left(Q Q^{\prime}\right)\right] e^{-\left(Q-Q^{\prime}\right)^{2} / 2}, \\
\zeta^{(1)}\left(q_{j}, q_{j^{\prime}}\right)=4 \pi \ell^{2} R^{2}\left[e^{-Q Q^{\prime}} I_{1}\left(Q Q^{\prime}\right)\right] e^{-\left(Q-Q^{\prime}\right)^{2} / 2}, \\
W_{j j^{\prime}}^{(0,1)}\left(\mathbf{q}, \mathbf{q}^{\prime}\right)=\frac{\hbar}{m^{2} L^{2}}\left(\frac{\pi j}{L}\right)^{2}\left(\frac{\pi j^{\prime}}{L}\right)^{2} \zeta^{(0,1)} .
\end{gathered}
$$

where $Q=q_{j} R, Q^{\prime}=q_{j^{\prime}} R$ (the corresponding equations for some other classes of the correlation functions can be found in Ref. 14).

Since $\theta$ and $t$ enter the matrix $\tilde{\gamma}_{j j^{\prime}}$ [Eq. (34)], only in the combination $\theta / t$ and only directly via the diagonal elements, the inversion of this matrix obviously makes $\chi_{\text {mix }}(\theta / t)$ a rational function of the polynomials of $\theta / t$. Since $\chi_{\text {mix }}(\theta / t=$ $0)=0$, such a structure of $\chi_{\text {mix }}(\theta / t)$ means that at $\theta / t \ll 1$

$$
\chi_{\text {mix }}(\theta / t \ll 1)=\frac{\theta}{t} V_{1}(u, L / a)+\frac{\theta^{2}}{t^{2}} V_{2}(u, L / a)+\cdots .
$$

These functions are illustrated in Figs. 5 and 6. Note that since $\theta$ contains a small parameter $\ell^{2}$ in denominator, the condition $\theta / t \ll 1$, necessary for the expansion (38), is much 


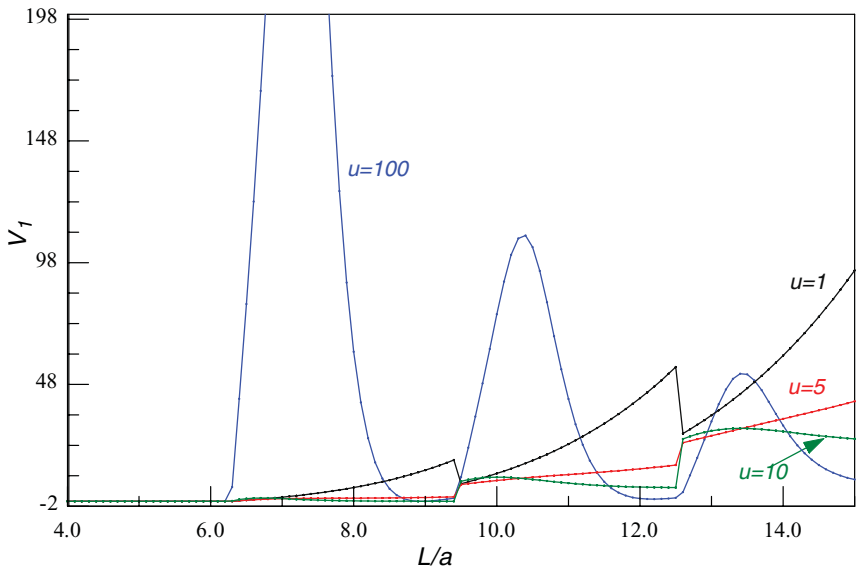

FIG. 5. (Color online) Coefficient $V_{1}$ [Eq. (38)] as a function of the film thickness $L / a$ for four values of $u: u=1$ (black), 5 (red), 10 (green), and 100 (blue). The curves are marked accordingly.

stronger than a simple condition of large bulk free paths $t \gg 1$ necessary for expansion (29) unless one deals with ultraclean films at ultralow temperatures for which $\alpha \ll 1$.

The condition $\theta / t \ll 1$, by itself, is not sufficient to justify expansion (38); the sufficient condition is $\theta / \phi t \ll 1$. This is a much stronger condition, especially because the coefficient $\phi$ [Eqs. (25) and (26)] rapidly decreases with an increase in $L / a$ or $u$ (Fig. 1). As is clear from Fig. 6, the coefficient $V_{2}$ rapidly increases with an increase in film thickness $L / a$ and the second term in the expansion (38) can easily overcome the first. However, in this case this is not a sign of crossover, as was the case for intrinsic interference. Expansion (38) in $1 / t$ for mixing interference is in reality an expansion in $\tau_{\mathrm{tr}}^{w} / \tau_{\mathrm{tr}}^{b}$ and the rapid growth of $V_{2}$ is simply a sign that these values of parameters the wall-driven transport time becomes much longer than the bulk one and the expansion fails. The mixing interference contribution $\chi_{\text {mix }}$ outside of domain (38) is illustrated in Fig. 7 for $\theta=0.1$ and $t=20$.

The existence of the mixing interference requires the presence of the off-diagonal terms in the collision operator $\widehat{\gamma}$, Eq. (15). If this whole matrix were of the $\delta$ type, as its

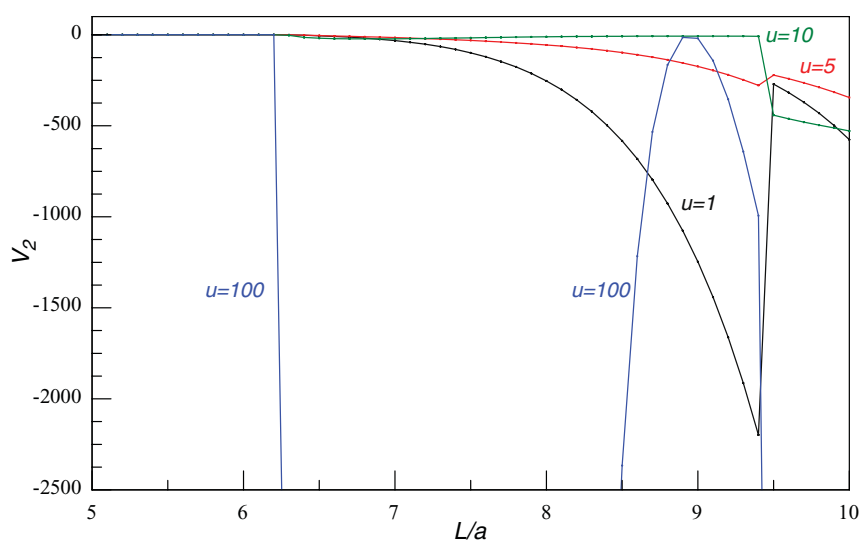

FIG. 6. (Color online) Coefficient $V_{2}$ [Eq. (38)] as a function of the film thickness $L / a$ for four values of $u: u=1$ (black), 5 (red), 10 (green), and 100 (blue). The curves are marked accordingly.

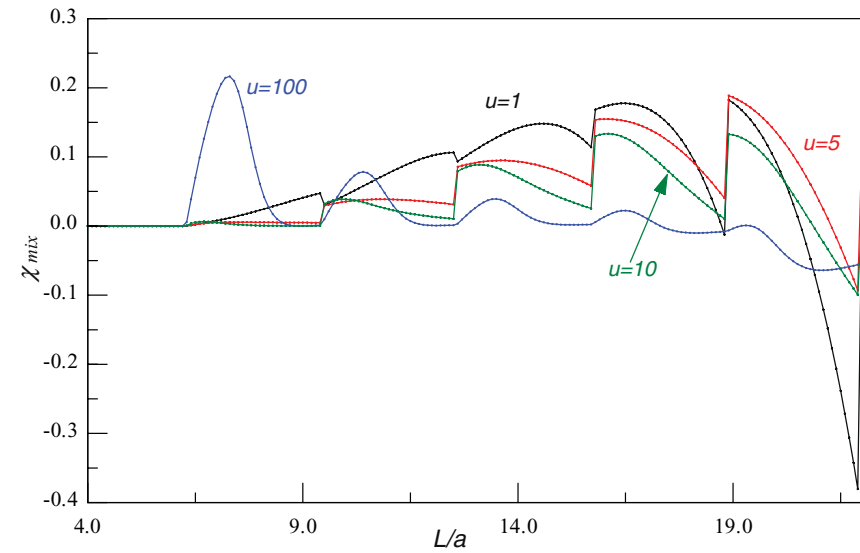

FIG. 7. (Color online) The mixing interference $\chi_{\text {mix }}$ [Eq. (33)] as a function of the film thickness $L / a$ for four values of $u: u=1$ (black), 5 (red), 10 (green), and 100 (blue). The curves are marked accordingly.

bulk-driven counterpart is assumed to be in this paper, the inversion would have been trivial and the intrinsic interference from the previous subsection would have been the only interference mechanism. The collision operator always has a $\delta$-type structure when there is only one active miniband, $S=1$. In this case $\chi_{\text {mix }}(S=1)=0$ exactly. This is confirmed in Figs. 5-7 at small values of $L / a$.

Outside of ultrathin films for which $S=1$, the matrix of the wall-driven scattering probabilities $\widehat{W}$ [Eqs. (13) and (14)] is indeed close to being diagonal at very large values of the correlation radius $R$ (large $u$ ) when the scatteringdriven change in momentum $\delta q \sim \hbar / R$ is too small to ensure transitions between the minibands. Still, even in this case one can observe a spontaneous, and very sharp, opening of transition channels $j \longleftrightarrow j+1$ when the film thickness reaches some critical value $L_{j}=\pi \sqrt{(j+1 / 2) a R / 2} \cdot{ }^{14} \mathrm{It}$ is instructive to analyze how this new type of quantum size effect not only results in a strong precipitous drop in conductivity ${ }^{14}$ but also leads to the re-emergence of the mixing interference.

At large $R$, when the thickness of the film approaches the value of $L_{1}$, the previously diagonal matrix $\widehat{\gamma}$ [Eq. (15)] acquires the first off-diagonal element,

$$
\gamma_{12}=\gamma_{21}=1 / \tau_{12}^{w} \text {. }
$$

The value of $\tau_{12}$ decreases exponentially when $L$ approaches $L_{1}$ and becomes practically equal to the wall-driven part of the diagonal elements $\gamma_{11,22}$ that in this case are approximately $1 / \tau_{\mathrm{tr}}^{w}$. The overall transport time also rapidly drops with a decrease in $\tau_{12}^{w}$ as

$$
\frac{1}{\tau_{\mathrm{tr}}} \sim \frac{\alpha}{\tau_{b}}+\frac{1}{\tau_{\mathrm{tr}}^{w}}-\frac{1}{\tau_{12}^{w}}-\frac{1}{\tau_{12}^{w 2}} \frac{\tau_{b} \tau_{\mathrm{tr}}^{w}}{\alpha \tau_{\mathrm{tr}}^{w}+\tau_{b}}=\frac{1}{\tau_{\mathrm{tr}}^{M}}-\frac{1}{\tau_{12}^{w}}-\frac{\tau_{\mathrm{tr}}^{M}}{\tau_{12}^{w 2}},
$$

meaning that the mixing interference grows by the absolute value starting from zero as

$$
\chi_{\mathrm{mix}}=-\frac{\tau_{\mathrm{tr}}^{M}}{\tau_{12}^{w}}\left(1+\frac{\tau_{\mathrm{tr}}^{M}}{\tau_{12}^{w}}\right)
$$




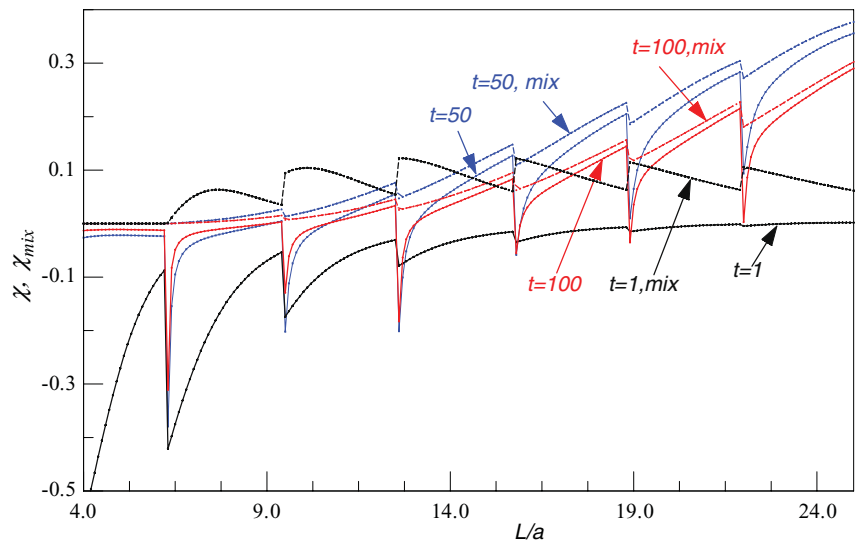

FIG. 8. (Color online) The full interference contribution $\chi$ (solid lines) and the mixing interference $\chi_{\text {mix }}$ (dashed lines denoted as mix) as a function of film thickness $L / a$ at $u=1, \theta=0.1$, and various values of $t: t=1$ (black), $t=50$ (blue), and $t=100$ (red). The curves are marked by the value of $t$.

until it reaches approximately

$$
\begin{aligned}
\chi_{\text {mix }} & \sim-\left(\frac{\alpha \tau_{\mathrm{tr}}^{w}}{\tau_{b}}+1\right)^{-2}\left(2+\frac{\alpha \tau_{\mathrm{tr}}^{w}}{\tau_{b}}\right) \\
& =-\left(\frac{\theta}{t \phi}+1\right)^{-2}\left(2+\frac{\theta}{t \phi}\right)
\end{aligned}
$$

at $\tau_{12}^{w} \sim \tau_{\mathrm{tr}}^{w}$. Equation (42) gives a good estimate of the mixing interference for walls with large-scale roughness $R \gg a$ and provides the "mixing" correction to the intrinsic interference for the gliding electrons (32).

\section{General results}

The above analysis of intrinsic and mixing interference can serve as a guide for study of interference effects in more general situations. For example, Fig. 8 represents a dependence of the relative interference contribution $\chi$ [Eq. (21)] on film thickness at various values of $t, t=1,50,100$, for films with small-scale roughness, $u=1$ and $\theta=0.1$. For comparison, we plotted in the same figure the relative mixing interference (dashed lines denoted by mix), $\chi_{\text {mix }}(L / a)$, for the same values of parameters. The difference between the solid and dashed curves reflects the contribution from intrinsic interference. Since the mixing interference is suppressed for single-band systems, i.e., at $L / a<2 \pi$, it is absolutely clear that the intrinsic interference dominates in ultrathin films and is gradually being replaced by the mixing interference with increasing thickness. Of course, since the value of $\theta$ here is not small, the mixing interference dominates in thick films. Interestingly, it looks like the intrinsic interference contribution is almost always negative while the mixing one is positive. The overall interference contribution tends to be destructive in very thin films and constructive in thicker ones.

Figure 9 extends the curves $\chi(L / a)$ and $\chi_{\text {mix }}(L / a)$ (also at $u=1$ and $\theta=0.1)$ to thicker films. It is clear that the interference contribution exhibits a maximum at certain thickness and that the positions of the maxima shift to the right with the increase in the bulk mean free path (with the increase in $t$ ). In very thick films, the bulk scattering

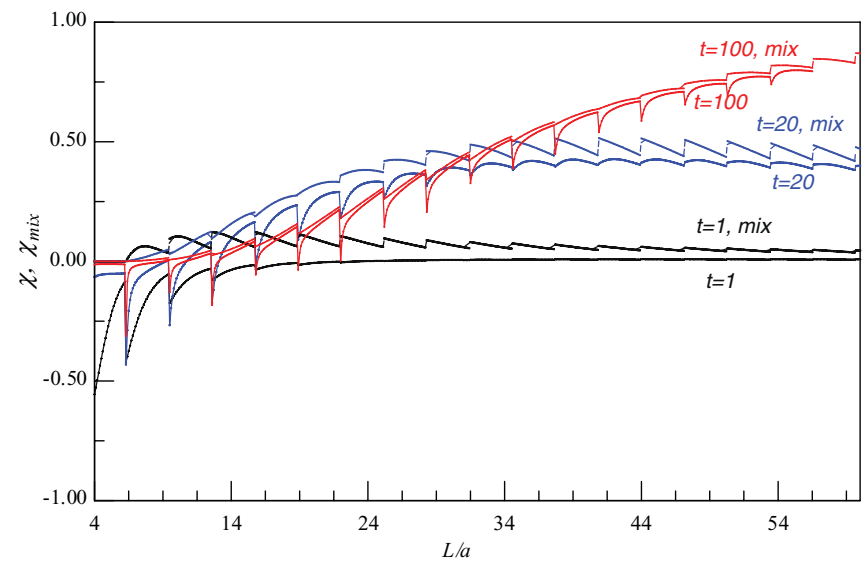

FIG. 9. (Color online) As described in the caption to Fig. 7 but for thicker films: $t=1$ (black), $t=20$ (blue), and $t=100$ (red).

obviously dominates the resistivity and all boundary processes, including the interference ones, become negligible, meaning that $\chi(L / a \rightarrow \infty) \rightarrow 0$.

The dependence of $\chi$ and $\chi_{\text {mix }}$ (dashed lines marked with the letter $m$ ) on $\theta=\alpha / \ell^{2}$ is illustrated in Fig. 10 at $t=20$ and $u=1$. Here, again, at $L / a<2 \pi$ we have a pure intrinsic interference, mostly destructive, which is gradually being replaced by the constructive mixing interference for thicker films. The replacement occurs faster at higher values of $\theta$. Interestingly, the sawtooth structure of the curves is much more pronounced for the overall and, therefore, intrinsic interference than for the mixing one. We do not have a simple explanation for this effect. The small values of $\theta$ for the curves in Fig. 10 should be interpreted as the consequences of small values of $\alpha$ rather than large values of $\ell / a$ : the computations were done at $u=1$ while our theory is valid at $\ell / a<\sqrt{u}$. If one wants to suppress $\theta$ by increasing the amplitude of inhomogeneities $\ell / a$, one should simultaneously increase the correlation radius of surface roughness $R / a \sim \sqrt{u}$.

Finally, Fig. 11 presents the curves $\chi(L / a)$ (solid lines) and $\chi_{\text {mix }}(L / a)$ (dashed lines marked as $m$ ) for $t=20$ and $\theta=0.1$

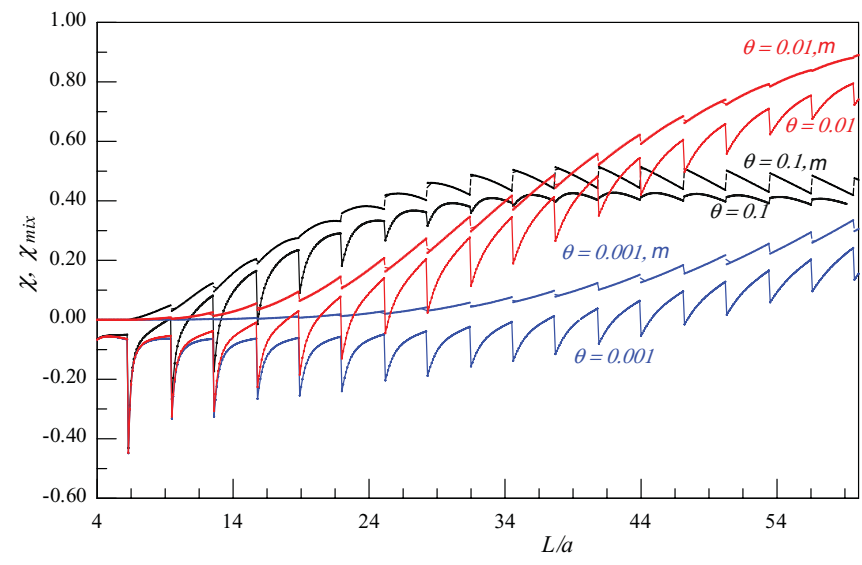

FIG. 10. (Color online) The full interference contribution $\chi$ (solid lines) and the mixing interference $\chi_{\text {mix }}$ (dashed lines marked as $m$ ) as a function of film thickness $L / a$ at $u=1, t=20$, and various values of $\theta: \theta=0.1$ (black), $\theta=0.01$ (red), and $\theta=0.001$ (blue). The curves are marked by the value of $\theta$. 


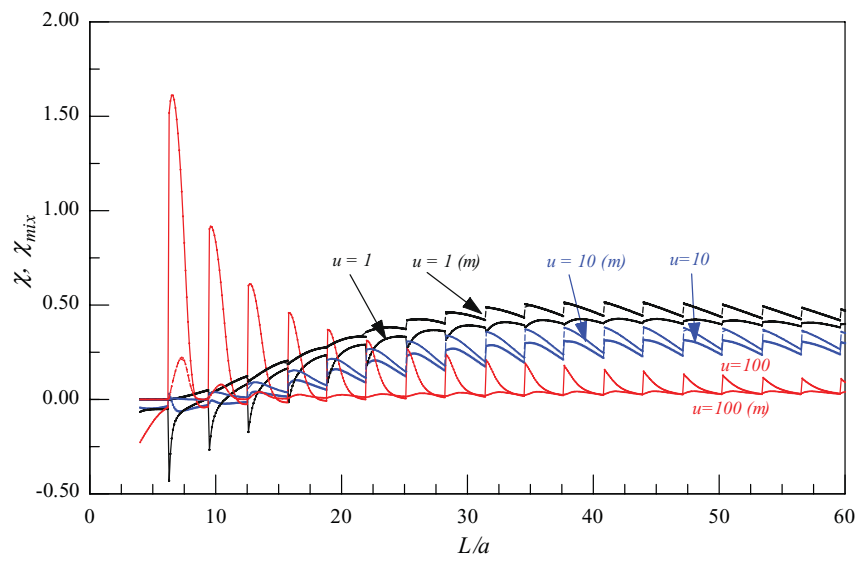

FIG. 11. (Color online) The full interference contribution $\chi$ (solid lines) and the mixing interference $\chi_{\text {mix }}$ (dashed lines marked as $m$ ) as a function of film thickness $L / a$ at $t=20, \theta=0.1$, and various values of $u: u=1$ (black), $u=10$ (blue), and $u=100$ (red). The curves are marked by the value of $u$.

at various values of $u, u=1,10,100$. The curves at $u=100$ differ from all the rest in that the contribution from intrinsic interference here seems to be positive. Also, the QSE of Ref. 14 is much more pronounced for the intrinsic and overall interference than for the mixing interference. This is easy to understand since this type of QSE practically never shows up when the bulk collisions present the dominant scattering channel (see the discussion in Ref. 14b).

\section{CONCLUSIONS}

In summary, we analyzed interference between bulk and surface scattering processes in resistivity of thin films in QSE conditions. General results can sometimes be not very transparent because of parameter clutter. The main parameters that affect the interference are the pure bulk transport time, the amplitude and the correlation radius of surface roughness, the film thickness, and the ratio of the bulk transport and collision times. In such a rich parameter space, the interference contributions can not only assume significantly different values but also even change their signs.

The uncertainty in some of the parameters, especially the values of the bulk transport and collision times in QSE conditions, makes comparison with experiment not easy. We highlighted the structure of desired experiments in the Introduction. There is currently one experimental group that measures the thin film resistivity simultaneously with the STM analysis of the surface (see Refs. 7,27,28,32,39 and references therein) though the experiments are done for thicker films, away from the QSE regime. Other groups ${ }^{8}$ have desirable QSE capabilities when dealing with STM of inhomogeneous buried interfaces but have not concentrated on measurements of conductivity. To the best of our knowledge, none of the experimental groups, with the exception of, maybe, Ref. 13, observed clear signs of QSE in resistivity, which makes a direct comparison of our results with experimental data in metals currently impossible. One of the options here is to average out the QSE gyrations and compare averaged data to experiment. We tried to follow this route with respect to the dependence of the resistivity on film thickness $\sigma(L)$. However, depending on the method of averaging of the same curve, the results for the transport time exhibited the functional dependence anywhere between $L^{-2.3}$ when using the tops of the peaks and $L^{-0.6}$ when using the minima. Most of the experimental curves, which also exhibit a large uncertainty (see discussion and references in Ref. 35), fit within this range. So far, the direct experimental confirmations of our quantum transport theory have been provided by nonmetal quantum systems. ${ }^{17,18}$

Our main emphasis was on revealing and following two distinct sources of such interference: the entanglement between the surface and bulk interaction lines in the self-energy of the single-particle Green's functions and the mixing resulting from the inversion of the collision operator in the generalized response function. We labeled these two types of interference in the paper as the intrinsic and mixing interference. The intrinsic and mixing interference lead to different types of behavior of non-Mathiessen's terms in resistivity of thin films. Earlier approaches included only the mixing interference (see Refs. 21 and 26-28 and references therein) though some of recent publications concentrated on intrinsic interference, ${ }^{36-38}$ without paying attention to the mixing one.

Generically, the mixing terms are larger than the intrinsic ones in thicker films. However, there are several important situations when the intrinsic interference clearly dominates. First, this happens in ultrathin films. When the thickness of the film $L$ is such that the QSE reduces the spectrum to a single miniband, $L / a<2 \pi$, the mixing interference is completely suppressed and only gradually picks up with increasing thickness. Second, since the intrinsic interference is built of the bulk collision time $\tau_{b}$ while the mixing one contains the bulk transport time $\tau_{\mathrm{tr}}^{b}$, the intrinsic interference can dominate when there is a considerable gap between these two times. This can happen, for example, in pure films at low temperatures when the bulk relaxation is due mainly to the phonon scattering. In this case the bulk transport time can exceed the collision one by a large factor $\left(T_{D} / T\right)^{2}$, resulting in the dominant role of the intrinsic interference effects.

We tried to compare our results with other quantum computations that explicitly include QSE. ${ }^{11,21,26,29}$ Most of earlier publications did not pay sufficient attention to the interference effects. References $21 \mathrm{a}$ and $21 \mathrm{~b}$ contain no explicit equations for the interference terms that can be compared with our results. The authors of Ref. 11 were not interested in the interference terms and considered bulk and roughness scattering as two independent additive channels. Reference 26 also contains no explicit information about the interference terms except for mentioning that these contributions seem to be smaller than the pure wall or bulk terms. As our results show, this is not always the case and the relative interference contribution $\chi$ can be quite large. In addition, Refs. 11,21, and 26 use the $\delta$-type (white noise) approximation for the surface roughness and, therefore, cannot describe the large-scale roughness and the corresponding crossover between different regimes.

In most of the parameter space, the contribution tends to be negative (destructive) from the intrinsic interference (thinner films) and positive (constructive) from the mixing one (thicker films). This qualitatively agrees with the experimental 
observation in Refs. 7,32, and 39. The quantitative comparison is currently impossible since there are no yet-confirmed observations of QSE in metals (see, e.g., Ref. 12), with the exception of Ref. 13, even before the transition to the ultraquantum regime with a complete destruction of the quasiclassical Fermi surface. ${ }^{40}$ In the Introduction we briefly discussed what kind of experiments could provide quantitative verification of our theory beyond the already-existing checks in experiments with quantized ultracold neutrons in rough waveguides ${ }^{17}$ and ${ }^{3} \mathrm{He}$ films. ${ }^{18}$
${ }^{1}$ A. A. Abrikosov, L. P. Gorkov, and I. E. Dzyaloshinski, Methods of Quantum Field Theory in Statistical Physics (Dover, New York, 1975).

${ }^{2}$ E. M. Lifshitz and L. P. Pitaevsky, Physical Kinetics, Course of Theoretical Physics Vol. IX, edited by L. Landau and E. M. Lifshitz (Pergamon, New York, 2002).

${ }^{3}$ J. Rammer and H. Smith, Rev. Mod. Phys. 58, 323 (1986).

${ }^{4}$ A. A. Abrikosov, Fundamentals of the Theory of Metals (NorthHolland, Amsterdam, 1988).

${ }^{5}$ J. J. Plombon, E. Andideh, V. M. Bubin, and J. Maiz, J. Appl. Phys. Lett. 89, 113124 (2006).

${ }^{6}$ H. Marom and M. Eizenberg, J. Appl. Phys. 99, 123705 (2006); T. Sun, B. Yao, A. P. Warren, K. Barmak, M. F. Toney, R. E. Peale, and K. R. Coffey, Phys. Rev. B 81, 155454 (2010).

${ }^{7}$ R. C. Munoz, C. Gonzalez, R. Henriquez, A. Espinosa, G. Kremer, L. Moraga, A. Ibanez-Landeta, S. Bahamondes, S. Donoso, and M. Flores, J. Appl. Phys. 110, 023710 (2011).

${ }^{8}$ I. B. Altfeder, D. M. Chen, and K. A. Matveev, Phys. Rev. Lett. 80, 4895 (1998); I. B. Altfeder, V. Narayanamurti, and D. M. Chen, ibid. 88, 206801 (2002); I. B. Altfeder, K. A. Matveev, and D. M. Chen, ibid. 78, 2815 (1997); F. Krok, F. Buatier de Mongeot, M. Goryl, J. J. Kolodziej, and M. Szymonski, Phys. Rev. B 81, 235414 (2010); Ying Jiang, Kehui Wu, Zhe Tang, Ph. Ebert, and E. G. Wang, ibid. 76, 035409 (2007).

${ }^{9}$ Ya. M. Blanter, M. I. Kaganov, A. V. Pantsulaya, and A. A. Varlamov, Phys. Rep. 245, 159 (1994); A. E. Meyerovich and D. Chen, Phys. Rev. B 66, 235306 (2002).

${ }^{10}$ V. B. Sandomirskii, Sov. Phys. JETP 25, 101 (1968) [Zh. Eksp. Teor. Fiz. 52, 158 (1968)].

${ }^{11}$ N. Trivedi and N. W. Ashcroft, Phys. Rev. B 38, 12298 (1988).

${ }^{12}$ G. Fischer and H. Hoffmann, Solid State Commun. 35, 793 (1980); G. Fischer, H. Hoffmann, and J. Vancea, Phys. Rev. B 22, 6065 (1980); L. A. Kuzik, Yu. E. Petrov, F. A. Pudonin, and V. A. Yakovlev, Sov. Phys. JETP 78, 114 (1994) [Zh. Exp. Teor. Fiz. 105, 215 (1994)].

${ }^{13}$ S. Farhangfar, Phys. Rev. B 76, 205437 (2007).

${ }^{14}$ A. E. Meyerovich and I. V. Ponomarev, Phys. Rev. B 65, 155413 (2002); Yiying Cheng, and A. E. Meyerovich, ibid. 73, 085404 (2006).

${ }^{15}$ M. Aono, APS Bulletin 47, 97 (2002); S. Hasegawa, I. Shiraki, and F. Tanabe, ibid. 98 (2002).

${ }^{16}$ U. Ramsperger, T. Uchihashi, and H. Nejoh, Appl. Phys. Lett. 78, 85 (2001).

${ }^{17}$ R. Adhikari, Y. Cheng, A. E. Meyerovich, and V. V. Nesvizhevsky, Phys. Rev. A 75, 063613 (2007).
${ }^{18}$ P. Sharma, A. Corcoles, R. G. Bennett, J. Parpia, B. Cowan, A. Casey, and J. Saunders, Phys. Rev. Lett. 107 (in print, 2011).

${ }^{19}$ E. H. Sondheimer, Adv. Phys. 1, 1 (1952); J. R. Sambles, Thin Solid Films 106, 321 (1983); K. Fuchs, Proc. Cambridge Philos. Soc. 34, 100 (1938).

${ }^{20}$ Y. Namba, Jpn. J. Appl. Phys. 9, 1376 (1970).

${ }^{21}$ Z. Tesanovic, M. V. Jaric, and S. Maekawa, Phys. Rev. Lett. 57, 2760 (1986); Z. Tesanovic, J. Phys. C 20, L289 (1987).

${ }^{22}$ A. E. Meyerovich and S. Stepaniants, Phys. Rev. Lett. 73, 316 (1994)

${ }^{23}$ G. Fishman and D. Calecki, Phys. Rev. Lett. 62, 1302 (1989); Phys. Rev. B 43, 11581 (1991).

${ }^{24}$ A. E. Meyerovich and A. Stepaniants, Phys. Rev. B 58, 13242 (1998); 60, 9129 (1999).

${ }^{25}$ D. Calecki, Phys. Rev. B 42, 6906 (1990).

${ }^{26}$ L. Sheng, D. Y. Xing, and Z. D. Wang, Phys. Rev. B 51, 7325 (1995).

${ }^{27}$ R. C. Munoz, G. Vidal, G. Kremer, L. Moraga, and C. Arenas, J. Phys. Condens. Matter 11, L299 (1999).

${ }^{28}$ R. C. Munoz, R. Finger, C. Arenas, G. Kremer, and L. Moraga, Phys. Rev. B 66, 205401 (2002).

${ }^{29}$ A. E. Meyerovich and A. Stepaniants, J. Phys. Condens. Matter 12, 5575 (2000).

${ }^{30}$ J. A. Ogilvy, Theory of Wave Scattering from Random Surfaces (Adam Hilger, Bristol, 1991).

${ }^{31}$ R. M. Feenstra, D. A. Collins, D. Z.-Y. Ting, M. W. Wang, and T. C. McGill, Phys. Rev. Lett. 72, 2749 (1994).

${ }^{32}$ R. C. Munoz, G. Vidal, G. Kremer, L. Moraga, C. Arenas, and A. Concha, J. Phys. Condens. Matter 12, 2903 (2000).

${ }^{33} \mathrm{G}$. Grimvall, The Electron/Phonon Interaction in Metals (Elsevier, Amsterdam, 1981).

${ }^{34}$ G. Kastle, H.-G. Boyen, A. Schroder, A. Plettl, and P. Ziemann, Phys. Rev. B 70, 165414 (2004).

${ }^{35}$ S. Chatterjee and A. E. Meyerovich, Phys. Rev. B 81, 245409 (2010).

${ }^{36}$ A. E. Meyerovich, J. Low Temp. Phys. 124, 461 (2001).

${ }^{37}$ R. M. Bowley and K. A. Benedict, J. Low Temp. Phys. 142, 701 (2006).

${ }^{38}$ B. Feldman, R. Deng, and S. Dunham, J. Appl. Phys. 103, 113715 (2008).

${ }^{39}$ R. C. Munoz, C. Arenas, G. Kremer, and L. Moraga, J. Phys. Condens. Matter 15, L177 (2003).

${ }^{40}$ A. E. Meierovich and B. E. Meierovich, Sov. Phys. JETP 66, 833 (1987) [Zh. Eksp. Teor. Fiz. 93, 1461 (1987)]; A. E. Meyerovich and D. Chen, Phys. Rev. B 66, 235306 (2002); Y. Ke, F. Zahid, V. Timoshevskii, K. Xia, D. Gall, and H. Guo, ibid. 79, 155406 (2009). 